TRANSACTIONS OF THE

AMERICAN MATHEMATICAL SOCIETY

Volume 288, Number 2, April 1985

\title{
PACKING MEASURE, AND ITS EVALUATION FOR A BROWNIAN PATH \\ BY
}

\section{S. JAMES TAYLOR AND CLAUDE TRICOT}

\begin{abstract}
A new measure on the subsets $E \subset \mathbf{R}^{d}$ is constructed by packing as many disjoint small balls as possible with centres in $E$. The basic properties of $\phi$-packing measure are obtained: many of these mirror those of $\phi$-Hausdorff measure. For $\phi(s)=s^{2} /(\log \log (1 / s))$, it is shown that a Brownian trajectory in $\mathbf{R}^{d}(d \geqslant 3)$ has finite positive $\phi$-packing measure.
\end{abstract}

1. Introduction. The size of sets of zero Lebesgue measure in $\mathbf{R}^{d}$ can be investigated by several distinct techniques. The first of these to be extensively developed was established by Hausdorff-for a recent account see Rogers [9]. Hausdorff measure is defined using economical covers of a set. For a given monotone function $\phi(s)$, there is a sense in which Hausdorff $\phi$-measure is the smallest outer measure determined by $\phi$; there are several other measures based on $\phi$. In this paper we define packing $\phi$-measure and examine some of its basic properties. We will see that it is larger than Hausdorff $\phi$-measure, and many of its properties mirror those of Hausdorff measure.

The definition of packing measure has to be given in two stages. In $\$ 3$ we consider various premeasures and eventually choose one of them to generate the $\phi$-packing measure $\phi-p(E)$ defined in $\S 5$. In the theory of Hausdorff measures, an essential tool used to prove $\phi-m(E)$ finite and positive is the use of upper density, first developed by Frostman [6] and made precise in [10]. The analogous theorem for packing measure, using lower density, is developed in $\$ 5$ and used for immediate calculations. For example, the classical Cantor set in $[0,1]$ has finite positive packing measure with respect to $\phi(s)=s^{\alpha}, \alpha=\log 2 / \log 3$, so that this set is regular in the sense that the correct $\phi$ giving finite positive measure is the same for both Hausdorff and packing measures.

The notion of strong and weak $\phi$-variations of a function $f:[a, b] \rightarrow \mathbf{R}^{d}$ was studied by Goffman and Loughlin in [7]. Just as the weak variation is related to Hausdorff measure, we are able to show, in $\$ 4$, that $\phi$-packing measure of the image of $f$ is bounded by the limiting strong $\phi$-variation. The strong variation of Brownian motion was obtained in [14], so this relationship gives an upper bound for the packing measure of a Brownian trajectory.

In [2] it was shown that $\phi(s)=s^{2} \log |\log s|$ is the correct function to give the Hausdorff measure of a Brownian trajectory in $\mathbf{R}^{d}(d \geqslant 3)$. In $\$ 7$ we show that

Received by the editors May 20, 1983 and, in revised form, June 8, 1984.

1980 Mathematics Subject Classification. Primary 28A12; Secondary 28A75, 60J65.

Key words and phrases. Packing measure, density theorem, Brownian motion, sojourn time.

(1)1985 American Mathematical Society $0002-9947 / 85 \$ 1.00+\$ .25$ per page 
$\psi(s)=s^{2} / \log |\log s|$ is the correct function to make the $\psi$-packing measure of the trajectory finite and positive. The proof requires a new analysis of the small values of the sojourn time in a ball for the process. This is carried out in $\S 6$ and leads to Theorem 6.8 which describes the lower asymptotic behaviour of the sojourn time. In order to set the scene we start in $\$ 2$ by developing notation and describing the main results for Hausdorff measure for which we can obtain analogues.

The set function $\phi-p(E)$ was first defined for subsets of $\mathbf{R}$ in [17, p. 145]. Sullivan [11] used the term "packing measure" for an outer measure $\nu_{p}$ which he defined on the subsets of a fixed closed set $\Lambda$ in $\mathbf{R}^{2}$. His measure $\nu_{p}$ depends critically on the local structure of $\Lambda$ and is not translation invariant. In general, $\phi-p \neq \nu_{p}$ when restricted to subsets of $\Lambda$ unless $\Lambda$ is sufficiently regular-for example, if $\Lambda$ were a rectifiable Jordan arc and $\phi(s)=s$.

2. Measures of Hausdorff type. We restrict our attention to subsets of Euclidean space $\mathbf{R}^{d}(d \geqslant 1)$. Some of our results extend to more general metric spaces, but we use the structure of $\mathbf{R}^{d}$ and do not concern ourselves with the validity of extensions. We use $|E|$ to denote the Lebesgue outer measure of $E$ and $\|x\|$ to denote the distance from 0 to $x \in \mathbf{R}^{d}$. We denote the open ball with centre at $x$ and radius $r>0$ by

$$
B_{r}(x)=\left\{y \in \mathbf{R}^{d}:\|x-y\|<r\right\}
$$

Let $\Gamma^{*}$ stand for the class of dyadic cubes in $\mathbf{R}^{d} . C \in \Gamma^{*}$ if it has side length $2^{-n}$, $n \in \mathbf{N}$, and each of its projections $\operatorname{proj}_{i} C$ on the $i$ th axis is a half-open interval of the form $\left[k_{i} 2^{-n},\left(k_{i}+1\right) 2^{-n}\right)$ with $k_{i} \in \mathbf{Z}$. For $x \in \mathbf{R}^{d}$, let $u_{n}(x)$ be the unique dyadic cube of side $2^{-n}$ containing $x$.

We also need the larger class $\Gamma^{* *}$ of semidyadic cubes. $C \in \Gamma^{* *}$ if it has side length $2^{-n}$ and $\operatorname{proj}_{i} C=\left[\frac{1}{2} k_{i} 2^{-n},\left(\frac{1}{2} k_{i}+1\right) 2^{-n}\right)$ with $k_{i} \in \mathbf{Z}$. Each $x \in \mathbf{R}^{d}$ belongs to $2^{d}$ cubes of side $2^{-n}$ in $\Gamma^{* *}$ : of these we denote by $v_{n}(x)$ the unique cube in $\Gamma^{* *}$ of side $2^{-n}$ whose complement is at distance $2^{-n-2}$ from $u_{n+2}(x)$. The coordinate of the centre of $v_{n}(x)$ is within $2^{-n-2}$ of the corresponding coordinate of $x$.

$\Phi$ denotes the class of functions $\phi:(0,1) \rightarrow \mathbf{R}$ which are increasing, continuous with $\phi(s) \rightarrow 0$ as $s \downarrow 0$, and satisfy a smoothness condition:

$$
\text { There exists } c_{0}>0 \text { such that } \phi(2 x)<c_{0} \phi(x) \text { for } 0<x<1 / 2 \text {. }
$$

The identity function $e(x)=x$, and each of its positive powers is clearly in $\Phi$.

Suppose $\mathscr{B}\left(\mathbf{R}^{d}\right)$ is the family of bounded subsets of $\mathbf{R}^{d}$. We introduce a partial order in the class of set functions $F: \mathscr{B}\left(\mathbf{R}^{d}\right) \rightarrow[0,+\infty]=\mathbf{R}^{+} \cup\{+\infty\}$. Put $F_{1} \preccurlyeq F_{2}$ if there is a finite $\lambda<0$ such that $F_{1}(E) \leqslant \lambda F_{2}(E)$ for all $E \in \mathscr{B}\left(\mathbf{R}^{d}\right)$, and write $F_{1} \asymp F_{2}$ and say $F_{1}$ and $F_{2}$ are equivalent if both $F_{1} \preccurlyeq F_{2}$ and $F_{2} \preccurlyeq F_{1}$.

$\operatorname{diam}(E)$ denotes the diameter of $E \in \mathscr{B}\left(\mathbf{R}^{d}\right)$. If $\mathscr{R} \subset \mathscr{B}\left(\mathbf{R}^{d}\right)$ put

$$
\phi(\mathscr{R})=\sum_{E \in \mathscr{R}} \phi(\operatorname{diam}(E)) \quad \text { and } \quad\|\mathscr{R}\|=\sup \{\operatorname{diam}(E): E \in \mathscr{R}\} .
$$


$\mathscr{F}\left(R^{d}\right)$ is a covering family if, for each $\varepsilon>0$, there is a subfamily $\mathscr{R} \subset \mathscr{F}$ with $\|\mathscr{R}\|<\varepsilon$ and $\mathbf{R}^{d} \subset \bigcup_{E \in \mathscr{R}} E$. Clearly this property is valid for $\Gamma^{*}$ or $\Gamma^{* *}$ or $\mathscr{S}$ the family of all open balls. For any covering family $\mathscr{F}$ define

$$
\mathscr{F} \phi-m(E)=\lim _{\varepsilon \downarrow 0} \inf \left\{\phi(\mathscr{R}):\|\mathscr{R}\|<\varepsilon, \mathscr{R} \subset \mathscr{F}, E \subset \bigcup_{F \in \mathscr{F}} F\right\} .
$$

Whenever $\mathscr{F}=\mathscr{B}\left(\mathbf{R}^{d}\right), \mathscr{F} \phi-m$ is the classical $\phi$-measure defined by Hausdorff, which we denote by $\phi-m$. When $\mathscr{F}=\mathscr{S}$ we obtain spherical Hausdorff measure. Since every bounded set is contained in a ball of double the diameter, (1) immediately implies

$$
\mathscr{S}_{\phi}-m \asymp \phi-m \text {. }
$$

When $\mathscr{F}=\Gamma^{*}$ we denote $\Gamma^{*} \phi-m$ by $\phi-m^{*}$, and again it is easy to see that

$$
\phi-m \asymp \phi-m^{*} .
$$

These restricted Hausdorff measures were first defined and used by Besicovitch. For our present purposes we make two further modifications. Given $E \subset \mathbf{R}^{d}$, let $\mathscr{S}_{E}$ be the family of open balls $\left\{B_{r}(x): r>0, x \in E\right\}$ with centres in $E$. We again get

$$
\phi-m(E) \leqslant \mathscr{S}_{E} \phi-m(E) \leqslant c \phi-m(E)
$$

for a suitable constant $c$. Now put

$$
\Gamma_{E}^{* *}=\left\{v_{n}(x): n \in \mathbf{N}, x \in E\right\},
$$

and denote $\Gamma_{E}^{* *} \phi-m(E)$ by $\phi-m^{* *}(E)$. It again follows that

$$
\phi-m \asymp \phi-m^{* *} \text {. }
$$

We note that each of the above set functions is defined on all subsets of $\mathbf{R}^{d}$, takes values in $[0,+\infty]$, and is a metric outer measure in the sense of Carathéodory. For each of them there is a class of measurable subsets $\mathscr{M}$, and the restriction of the set function to $\mathscr{M}$ is a $\sigma$-additive measure. Further, $\mathscr{M}$ contains the Borel subsets of $\mathbf{R}^{d}$. The rarefaction index corresponding to Hausdorff measure is usually called the Hausdorff-Besicovitch dimension and is defined by

$$
\operatorname{dim} E=\inf \left\{a>0: e^{a}-m(E)=0\right\}=\sup \left\{a>0: e^{a}-m(E)=+\infty\right\} .
$$

Clearly (3)-(6) show that any of the above set functions would have given the same value of $\operatorname{dim} E$. However if $\operatorname{dim} E=\delta>0$, then $e^{\delta}-m(E)$ can be zero, finite and positive, or $+\infty$. Only if $0<e^{\delta}-m(E)<+\infty$ do we say that $\phi(s)=s^{\delta}$ is the correct function in $\Phi$ for giving the Hausdorff measure of $E$.

If we want to prove that $\phi-m(E)<+\infty$, it is sufficient to find $K<+\infty$ such that for each $\varepsilon>0$ there is a cover $\mathscr{R}$ of $E$ for which $\|\mathscr{R}\|<\varepsilon$ and $\phi(\mathscr{R}) \leqslant K$. By using definition (2), it is harder to show that $\phi-m(E)>0$, for now we have to find $c>0$ such that $\phi(\mathscr{R}) \geqslant c$ for every possible cover of $E$. To avoid the need to consider all covers, the following density theorem, which is immediately deducible from the results in [10], is a useful tool. 
THEOREM 2.1. For a given $\phi \in \Phi$ there are constants $c_{1}, c_{2}$ such that, for all $E \subset \mathbf{R}^{d}$ and every finite Borel measure $\mu$ in $\mathbf{R}^{d}$,

$$
\begin{aligned}
c_{1} \mu(E) \inf _{x \in E} & \left\{\liminf _{n \rightarrow \infty} \frac{\phi\left(2^{-n}\right)}{\mu\left[u_{n}(x)\right]}\right\} \\
& \leqslant \phi-m(E) \leqslant c_{2} \mu\left(\mathbf{R}^{d}\right) \sup _{x \in E}\left\{\liminf _{\substack{\varepsilon \downarrow 0 \\
\operatorname{diam}(u) \leqslant \varepsilon}} \frac{\phi(d(u))}{\mu(u)}\right\},
\end{aligned}
$$

where the last infimum is taken over nondegenerate rectangles $u$ containing $x$ with diameter at most $\varepsilon$.

Variants of this theorem can be obtained by using (3)-(6) to replace $\phi-m$ by one of the restricted measures and by replacing the lim inf by

$$
\liminf _{\varepsilon \downarrow 0} \frac{\phi(\varepsilon)}{\mu\left(B_{\varepsilon}(x)\right)} \quad \text { or } \quad \liminf _{n \rightarrow \infty} \frac{\phi\left(2^{-n}\right)}{\mu\left[v_{n}(x)\right]} .
$$

A necessary tool for examining Brownian paths will be a density theorem for packing measure. Our choice of precise definition will ensure that such a density theorem is valid. We would point out that, in terms of a given $\phi \in \Phi$, there are other measures which lie between $\phi-m$ and $\phi-p$. No density theorem is known for these, and in some cases one can show that none is valid.

We use $K$ to denote a finite positive constant whose value is unimportant and may change from line to line.

3. Packing premeasures. Before defining new outer measures ( $\$ 5)$, our first step is to consider several ways of modifying definition (2) by changing inf to sup and replacing covers of $E$ by packings of disjoint sets related to $E$. We again assume $\mathscr{F}$ is a covering family of bounded sets in $\mathbf{R}^{d}$. We say $\mathscr{R} \subset \mathscr{B}\left(\mathbf{R}^{d}\right)$ is a packing of $E$ if, for all $F \in \mathscr{R}, \bar{E} \cap \bar{F} \neq \varnothing$ and the sets in $\mathscr{R}$ are disjoint. Put

$$
\mathscr{F} \phi-\mathscr{P}(E)=\limsup _{\varepsilon \downarrow 0}\{\phi(\mathscr{R}):\|\mathscr{R}\| \leqslant \varepsilon, \mathscr{R} \subset \mathscr{F}, \mathscr{R} \text { is a packing of } E\} .
$$

If we made no restriction on $\mathscr{F}$, this would not be a useful definition, because near any $x \in E$ one could fit in an arbitrary number of disjoint sets of diameter $\varepsilon>0$ having $x$ in their closure. This would give a value $+\infty$ even for the singleton $\{x\}$. In [19] definition (7) was used with $\mathscr{F}=$ the class of open balls. That definition is now denoted

$$
\mathscr{F} \phi-\mathscr{P}(E)=\phi-Q(E) .
$$

Different natural choices of $\mathscr{F}$ yield premeasures and measures which are not equivalent, although we will see that they all lead to the same rarefaction index $\Delta$ discussed in [18].

We consider three further possibilities for $\mathscr{F}$.

(i) $\mathscr{S}_{E}$ is the family of balls $B_{r}(x) r>0, x \in E . \mathscr{S}_{E} \phi-\mathscr{P}(E)$ is denoted by $\phi-P(E)$.

(ii) $\Gamma_{E}^{*}=\left\{u_{n}(x): n \in \mathbf{N}, x \in E\right\} \subset \Gamma^{*} . \Gamma_{E}^{*} \phi-\mathscr{P}(E)$ is denoted by $\phi-P^{*}(E)$. 
(iii) $\Gamma_{E}^{* *}=\left\{v_{n}(x): n \in \mathbf{N}, x \in E\right\} \subset \Gamma^{* *}$. $\Gamma_{E}^{* *} \phi-\mathscr{P}(E)$ is denoted by $\phi-$ $P^{* *}(E)$.

We discard $\phi-Q$ and $\phi-P^{*}$ for reasons which later become evident and show that $\phi-P$ and $\phi-P^{* *}$ are equivalent set functions. $\phi-P^{* *}$ will be useful as an auxiliary set function, but our final definition will be based on $\phi-P$. The first step is to clarify the simple properties of the set functions based on (7), which we have defined.

LEMMA 3.1. Let $\tau=\tau_{\phi}$ be one of the set functions $\phi-P, \phi-P^{*}, \phi-P^{* *}, \phi-Q$. Then

(i) $\tau$ is monotone: $E_{1} \subset E_{2} \Rightarrow \tau\left(E_{1}\right) \leqslant \tau\left(E_{2}\right)$.

(ii) $\tau$ is subadditive: $\tau\left(E_{1} \cup E_{2}\right) \leqslant \tau\left(E_{1}\right)+\tau\left(E_{2}\right)$, with equality holding if the distance between $E_{1}$ and $E_{2}$ is positive.

(iii) If $\phi(s) / \psi(s) \rightarrow 0$ as $s \rightarrow 0$, then $\tau_{\psi}(E)<+\infty \Rightarrow \tau_{\phi}(E)=0$.

(iv) $\phi-m \preccurlyeq \tau_{\phi}$.

(v) If $E=\{x\}$, then $\tau_{\phi}(E)=0$.

(vi) If $E$ is a bounded Lebesgue measurable subset of $\mathbf{R}^{d}$ with positive Lebesgue measure and $\phi(s)=s^{d}$, then $0<\tau_{\phi}(E)<+\infty$.

Proof. Most of these properties are immediate consequences of the definition of $\tau$. We note that (ii) follows from the fact that any packing $\mathscr{R}$ of $E_{1} \cup E_{2}$ can be separated into a packing of $E_{1}$ and a packing of $E_{2}$ with no interference when $\|\mathscr{R}\|$ is smaller than half the distance between $E_{1}$ and $E_{2}$. (iv) is easily verified for $\phi-P$ : if $M_{r}(E)$ denotes the maximum number of disjoint balls of radius $r$ centered in $E$, then

$$
\phi-P(E) \geqslant \underset{r \rightarrow 0}{\limsup } M_{r}(E) \phi(2 r),
$$

while $E$ can be covered by $M_{r}(E)$ balls of radius $2 r$, so that

$$
\phi-m(E) \leqslant \liminf _{r \rightarrow 0} M_{r}(E) \phi(4 r) .
$$

The same holds for the other $\tau_{\phi}$, with an analogous proof, or the use of the following Lemma 3.4. The lower bound of (vi) derives directly from (iv). The upper bound is evident.

REMARK. All the above are true for Hausdorff measure and its variants. For Hausdorff measure (ii) can be strengthened to $\tau\left(\bigcup_{i=1}^{\infty} E_{i}\right) \leqslant \sum_{i=1}^{\infty} \tau\left(E_{i}\right)$. This is false in the present case. Consider $\phi(s)=s^{1 / 2}$ and $E=Q \cap[0,1]$, the set of rationals in $[0,1]$. Since $\tau_{\phi}(E)=\tau_{\phi}([0,1]), \tau_{\phi}(E)=+\infty$, but $E$ is a countable union of singletons. Thus $\tau$ is not an outer measure.

LEMMA 3.2. If $\tau$ is as above, there exists for all $E \in \mathscr{B}\left(\mathbf{R}^{d}\right)$ a Borel set $B \supset E$ such that $\tau(B)=\tau(E)$. For $\phi-P$ or $\phi-Q$ we can take $B=\bar{E}($ closure of $E)$.

Proof. Any packing of $E$ is a packing of $\bar{E}$ and, conversely, so that $\phi-Q(\bar{E})=$ $\phi-Q(E)$. Given $\varepsilon>0$ and a family $\mathscr{R}$ of disjoint open balls of radii $r_{k}$ and centre $x_{k} \in \bar{E}$, we can always find a new family $\mathscr{R}^{\prime}$ of balls of radii $r_{k}^{\prime}$ and centre $x_{k}^{\prime} \in E$ such that $B_{r_{k}^{\prime}}\left(x_{k}^{\prime}\right) \subset B_{r_{k}}\left(x_{k}\right)$, and $\sum \phi\left(2 r_{k}\right) \leqslant(1+\varepsilon) \sum \phi\left(2 r_{k}^{\prime}\right)$. This gives $\phi-P(\bar{E}) \leqslant$ $(1+\varepsilon) \phi-P(E)$ for all $\varepsilon>0$. The monotonicity of $\phi-P$ gives the opposite 
inequality: $\phi-P(E) \leqslant \phi-P(\bar{E})$. For $\phi-P^{*}$ take the Borel set $B=\bigcap_{n} \cup\left\{u_{n}(x)\right.$ : $x \in E\})$ which contains $E$ : since $\Gamma_{B}^{*}=\Gamma_{E}^{*}$, we get $\phi-P^{*}(B)=\phi-P^{*}(E)$. The same argument works for $\phi-P^{* *}$.

The next lemma is the main reason we discard $\phi-Q$ as a candidate for packing premeasure:

Lemma 3.3. Let $\phi(s) \in \Phi$ be such that $s / \phi(s) \in \Phi$, and let $E$ be an infinite set included in $[0,1]$. Then $\phi-Q(E)=0$ or $+\infty$.

Functions satisfying such conditions are common: for example $s^{\alpha}, 0<\alpha<1$.

Proof. If $|\bar{E}|>0$ we use the fact that $s^{-1} \phi(s) \rightarrow+\infty$ and Lemmas 3.1(iii), (vi), and 3.2 to find $\phi-Q(E)=+\infty$.

If $|\bar{E}|=0$, let us write $\bar{E}$ as $[0,1]-\cup_{1}^{\infty} J_{i}$ where the $J_{i}$ are disjoint open intervals of decreasing lengths. There are two cases: either $\sum \phi\left(\left|J_{i}\right|\right)$ diverges: using packings made up of the $J_{i}$ we deduce that $\phi-Q(E)=+\infty$; or $\sum \phi\left(\left|J_{i}\right|\right)$ converges: let $s_{0}>0$ be such that $s^{-1} \phi(s)$ is decreasing on $] 0, s_{0}\left[\right.$. For all sequences $\left(a_{n}\right)$ of positive reals such that $\sum a_{n}<s_{0}$, we have $\phi\left(\sum a_{n}\right) \leqslant \sum \phi\left(a_{n}\right)$. Now let $0<\varepsilon<s_{0}$, and let $\mathscr{R}$ be a packing of $E$ by disjoint intervals of length $\leqslant \varepsilon$. If $u \in \mathscr{R}$, the fact that $|\bar{E}|=0$ implies

$$
|u|=\sum\left\{\left|J_{i} \cap u\right|: J_{i} \cap u \neq \varnothing\right\}
$$

so that

$$
\phi(|u|) \leqslant \sum\left\{\phi\left(\left|J_{i} \cap u\right|\right): J_{i} \cap u \neq \varnothing\right\} .
$$

Let $N(\varepsilon)$ be the largest integer $n$ such that $\left|J_{n}\right|>\varepsilon$. Adding up the last inequality for all $u \in \mathscr{R}$, we see that the same $J_{i}$ is never counted more than twice, and that if $i \leqslant N(\varepsilon)$, its contribution to the total sum is less than $2 \phi(\varepsilon)$. Therefore

$$
\begin{aligned}
\frac{1}{2} \phi(\mathscr{R}) & \leqslant \sum_{N(\varepsilon)}^{\infty} \phi\left(\left|J_{i}\right|\right)+N(\varepsilon) \phi(\varepsilon) \\
& \leqslant \sum_{N(\varepsilon)}^{\infty} \phi\left(\left|J_{i}\right|\right)+\sum_{N(\eta)}^{\infty} \phi\left(\left|J_{i}\right|\right)+N(\eta) \phi(\varepsilon)
\end{aligned}
$$

if $\eta$ is any number $>\varepsilon$. Letting $\varepsilon \rightarrow 0$ and then $\eta \rightarrow 0$, we see that the right member tends to 0 , and therefore $\phi-Q(E)=0$.

LEMMA 3.4. $\phi-P \asymp \phi-P^{* *} \preccurlyeq \phi-P^{*}$ and $\phi-P \preccurlyeq \phi-Q$.

Proof. From the definition of $v_{n}(x)$ it follows that $B_{2^{-n-2}}(x) \subset v_{n}(x) \subset B_{\rho 2^{-n}}(x)$, where $\rho=d^{1 / 2}$ is the diameter of a unit cube. Condition (1) now implies the first equivalence $\phi-P \asymp \phi-P^{* *}$. Since every semidyadic $v_{n}(x)$ contains the dyadic $u_{n+1}(x)$, we get $\phi-P^{* *} \preccurlyeq \phi-P^{*}$. The last inequality is obvious.

EXAMPLE 3.5 . Let $E_{0}$ be the perfect symmetric set in $[0,1]$ with ratio $1 / 4$. That is,

$$
E_{0}=\left\{x \in \mathbf{R}: x=\sum a_{n} 4^{-n}, a_{n}=0 \text { or } 3\right\} .
$$

The family of all dyadic intervals $u=u_{n}(x)$ can be divided in two classes $\mathscr{C}_{1}, \mathscr{C}_{2}$, according as $\dot{u} \cap E_{0} \neq \varnothing$ or $\dot{u} \cap E_{0}=\varnothing$. In the last case, $u$ has the form $u_{n}\left(k 2^{-n}\right)$. 
If $\mathscr{R}$ is any packing of $E_{0}$ made up of intervals of $\mathscr{C}_{1}, e^{1 / 2}(\mathscr{R})$ is at most equal to 1 and actually equal to 1 in the case where $\mathscr{R}$ covers all of $E_{0}$ except for a countable subset. Therefore, $\mathscr{C}_{1} e^{1 / 2}-\mathscr{P}\left(E_{0}\right)=1$.

Given $\varepsilon<1, x \in E_{0}$, there is a $u \in \mathscr{C}_{1}$ such that $u \subset B_{\varepsilon}(x)$ and $|u| \geqslant \varepsilon / 2$. It follows that

$$
e^{1 / 2}-P\left(E_{0}\right) \leqslant c_{0}^{2} \mathscr{C}_{1} e^{1 / 2}-\mathscr{P}\left(E_{0}\right)<+\infty .
$$

Since $\mathscr{C}_{2}$ contains, for all $n, 2^{n}-1$ disjoint intervals of length $2^{-2 n}$, we deduce, using these packings, that $e^{1 / 2}-P^{*}\left(E_{0}\right)=+\infty$. Therefore, $\phi-P$ and $\phi-P^{*}$ are not equivalent.

But if $E_{1}$ is defined as $E_{0}$ less the countable set of endpoints of the complementary intervals, $\mathscr{C}_{2}$ is empty and $e^{1 / 2}-P^{*}\left(E_{1}\right)<+\infty$. However, $e^{1 / 2}-Q\left(E_{1}\right)=+\infty$. Therefore, $\phi-P^{*}$ is not equivalent to $\phi-Q$. This example also shows that $\phi-P^{*}(E)$ is not always equal to $\phi-P^{* *}(E)$.

REMARK 3.6. In the light of this example, the reader may feel that the definition of $\phi-P^{*}$ is artificial because it depends too heavily on the presence of the dyadic points in the set. If, instead, we were to require the packing dyadic cubes to have an interior point on $E$, we would get a smaller premeasure, defined only on subsets of $\mathbf{R}^{d}-D$, where $D$ is the set of points with at least one coordinate of the form $k 2^{-n}$. It would still be rejected because of the undesirable properties of $\phi-p^{*}$ (see Example 5.7). The definition given for $\phi-P^{*}$ yields an easy proof for the density theorem 5.4 .

In [13] we noted that the gap between $\phi-m$ and generalised $\psi$-capacity in the sense of Frostman [6] was about a factor $|\log s|$. The same phenomenon exhibits itself concerning the maximum 'gap' between $\phi-P, \phi-P^{*}$, and $\phi-Q$. We now make this precise:

LemMA 3.7. Let $g$ : $] 0,1\left[\rightarrow \mathbf{R}^{+}\right.$be such that $\Sigma 1 / g\left(2^{-n}\right)<+\infty$ and let $\phi \in \Phi$ be such that $\phi / g=\psi \in \Phi$. Then $\phi-P(E)<+\infty \Rightarrow \psi-Q(E)=0$.

Proof. Let $E \in \mathscr{B}\left(\mathbf{R}^{d}\right)$. We recall that $M_{r}(E)$ denotes the maximum number of disjoint open balls of diameter $r$ centered in $E$. Since $\phi-P(E)<+\infty$, there exists $K<+\infty$ such that $\sup _{n} M_{2^{-n}}(E) \phi\left(2^{-n}\right) \leqslant K$. There exists also a constant $L_{d}$ such that no ball of radius $2^{-n}$ is at distance $\leqslant 2^{-n}$ from $L_{d}$ disjoint balls of radius $\geqslant 2^{-n}$. Let $N \in \mathbf{N}$, and let $\mathscr{R}$ be a family of disjoint balls at distance 0 from $E,\|\mathscr{R}\| \leqslant 2^{-N-2}$. Let $k_{n}$ be the number of balls in $\mathscr{R}$ of radius between $2^{-n}$ and $2^{-n+1}, n \geqslant N$. Since $k_{n} \leqslant L_{d} M_{2^{-n}}(E)$, we have

$$
\psi(\mathscr{R}) \leqslant \sum_{N}^{\infty} k_{n} \psi\left(2^{-n+1}\right) \leqslant c_{0} K L_{d} \sum_{N}^{\infty} \frac{1}{g\left(2^{-n}\right)},
$$

which tends to 0 as $N$ tends to $+\infty$. Hence, $\psi-Q(E)=0$.

Using any of the above definitions we could use the normal technique to obtain a rarefaction index. The following result shows that we always get the same index: 
COROllary 3.9. There is an index $\Delta$ such that for all $E \in \mathscr{B}\left(\mathbf{R}^{d}\right)$ :

(i) if $0<\delta<\Delta(E)$,

$$
e^{\delta}-P(E)=e^{\delta}-P^{*}(E)=e^{\delta}-P^{* *}(E)=e^{\delta}-Q(E)=+\infty .
$$

(ii) if $\delta>\Delta(E)$,

$$
e^{\delta}-P(E)=e^{\delta}-P^{*}(E)=e^{\delta}-P^{* *}(E)=e^{\delta}-Q(E)=0 .
$$

From Lemma 3.1, $\Delta$ is such that

$$
\operatorname{dim}(E) \leqslant \Delta(E) \leqslant d .
$$

For subsets of $\mathbf{R}$ there are many other equivalent definitions of $\Delta$ (see Tricot [18]).

4. Relation with strong variation. Suppose $f: I=[0,1] \rightarrow \mathbf{R}^{d}$ and $\pi$ is a dissection of $[0,1]$,

$$
0=x_{0}<x_{1}<\cdots<x_{n}=1, \quad \sigma(\pi)=\max \left(x_{i}-x_{i-1}\right) .
$$

Define the strong $\varphi$-variation $W_{\phi}(f)$ and limiting strong $\phi$-variation $V_{\phi}(f)$ by

$$
\begin{gathered}
W_{\phi}(f)=\sup _{\pi} \sum_{i=1}^{n} \phi\left(\left\|f\left(x_{i}\right)-f\left(x_{i-1}\right)\right\|\right), \\
V_{\phi}(f)=\lim _{\varepsilon \downarrow 0} \sup _{\sigma(\pi)<\varepsilon} \sum_{i=1}^{n} \phi\left(\left\|f\left(x_{i}\right)-f\left(x_{i-1}\right)\right\|\right) .
\end{gathered}
$$

There is an easy connection between $V_{\phi}(f)$ and $\phi-P(f(I))$. The following proof is due to the referee-it replaces our earlier more complicated version.

Theorem 4.1. Suppose $f: I \rightarrow \mathbf{R}^{d}$ is continuous and $\phi \in \Phi$. Then $\phi-P(f(I)) \leqslant$ $2 c_{0} V_{\phi}(f)$.

Proof. Given $\eta>0$, choose $\varepsilon>0$ such that

$$
\sup _{\sigma(\pi)<\varepsilon} \sum_{1}^{n} \phi\left(\left\|f\left(x_{i}\right)-f\left(x_{i-1}\right)\right\|\right)<V_{\phi}(f)+\eta .
$$

Now choose $\delta>0$ such that $\phi(\delta)=\varepsilon^{2}$. Let $\mathscr{R}$ be a finite disjoint family of balls $B_{i}$ of diameter less than $\delta$ and centres $f\left(x_{i}\right)$ with $\left.x_{i}<x_{i+1}, x_{i} \in\right] 0,1[$. Together with 0 and 1 the points $x_{i}, 0 \leqslant i \leqslant n$, form a dissection $\pi$ of $[0,1]$. Form the subcollection $\mathscr{R}_{1}$ of balls centered at $f\left(x_{i}\right)$ such that $x_{i}-x_{i-1} \geqslant \varepsilon$ and $\mathscr{R}_{2}=\mathscr{R} \backslash \mathscr{R}_{1}$. Since the number of balls in $\mathscr{R}_{1}$ is at most $\varepsilon^{-1}$, we get

$$
\phi\left(\mathscr{R}_{1}\right) \leqslant \varepsilon^{-1} \phi(\delta)=\varepsilon .
$$

Add further division points to the dissection $\pi_{2}$ formed from the $x_{i}$ such that $f\left(x_{i}\right)$ is a centre of a ball in $\mathscr{R}_{2}$, to give a new dissection $\pi_{3}$, with $\sigma\left(\pi_{3}\right)<\varepsilon$, containing no extra division point in $] x_{i-1}, x_{i}\left[\right.$ if $x_{i} \in \pi_{2}$. Now

$$
\operatorname{diam} B_{i}<2\left\|f\left(x_{i}\right)-f\left(x_{i-1}\right)\right\|,
$$


so, by (1),

$$
\phi\left(\operatorname{diam} B_{i}\right) \leqslant 2 c_{0} \phi\left(\left\|f\left(x_{i}\right)-f\left(x_{i-1}\right)\right\|\right)
$$

and

$$
\begin{aligned}
\phi\left(\mathscr{R}_{2}\right) & \leqslant 2 c_{0} \sum_{x_{i} \in \pi_{3}} \phi\left(\left\|f\left(x_{i}\right)-f\left(x_{i-1}\right)\right\|\right) \\
& \leqslant 2 c_{0}\left(V_{\phi}(f)+\eta\right) \quad \text { by }(11) .
\end{aligned}
$$

Therefore, $\phi(\mathscr{R}) \leqslant \varepsilon+2 c_{0}\left(V_{\phi}(f)+\eta\right)$. If $\eta$ and $\varepsilon$ tend to $0, \delta$ tends also to 0 , so that the theorem is proved.

This theorem is not, in general, sharp. The gap between critical functions $\phi$ for $\phi-P(f(I))$ and $V_{\phi}(f)$ can be arbitrarily large.

EXAMPLE 4.2. Suppose $\phi$ is any strictly increasing continuous function. Define $f$ : $I \rightarrow \mathbf{R}$ by

$$
f(0)=0, f(x)=\phi^{-1}(x) \sin (1 / x) \quad \text { for } 0<x \leqslant 1 .
$$

Take $z_{k}=2 / k \pi, k=1,2, \ldots$, to see that $V_{\phi}(f)=+\infty$. However, $f(I)$ is a bounded interval so $e-P(f(I))<+\infty$.

If we want $f$ to be bijective we could take the graph of the above $f$ in $\mathbf{R}^{2}$.

5. Packing measure. We now return to the premeasures $\tau$ which are the subject of Lemma 3.1 and use Method I of Munroe [8, Theorem 11.3] to define an outer measure

$$
\mu(E)=\inf \left\{\sum \tau\left(E_{n}\right): E_{n} \in \mathscr{B}\left(\mathbf{R}^{d}\right), E \subset \cup E_{n}\right\}
$$

defined for all subsets $E$ of $\mathbf{R}^{d}$. Let us first summarize some of the properties of $\mu$ :

LEMMA 5.1. If $\tau$ is one of the premeasures $\phi-P, \phi-P^{*}, \phi-P^{* *}, \phi-Q$, the outer measure $\mu$ defined by (12) is such that:

(i) $\mu$ is metric: $\operatorname{dist}(E, F)>0 \Rightarrow \mu(E \cup F)=\mu(E)+\mu(F)$.

(ii) All Borel sets in $\mathbf{R}^{d}$ are $\mu$-measurable.

(iii) $\mu$ is Borel regular: for all $E \subset \mathbf{R}^{d}$ there is a Borel set $B \supset E$ such that $\mu(B)=\mu(E)$.

(iv) If $E \in \mathscr{B}\left(\mathbf{R}^{d}\right), \mu(E) \leqslant \tau(E)$.

(v) For any sequence of sets $E_{n} \uparrow E, \mu\left(E_{n}\right) \rightarrow \mu(E)$.

(vi) If $E$ is $\mu$-measurable, $0<\mu(E)<\infty$ and $\varepsilon>0$, there exists a closed set $F \subset E$ such that $\mu(F)>\mu(E)-\varepsilon$.

(vii) For any $E$,

$$
\mu(E)=\inf \left\{\lim \tau\left(E_{n}\right): E_{n} \in \mathscr{B}\left(\mathbf{R}^{d}\right), E_{n} \uparrow E\right\} .
$$

Proof. (i) comes from the fact that for any $\varepsilon>0$ we can take the sets $E_{n}$ in (12) to be included in $\bigcup_{x \in E} B_{\varepsilon}(x)$. Then apply Lemma 3.1(ii). Property (ii) of Lemma 5.1 is true for any metric outer measure [8]. (iii) comes from Lemma 3.2: we can assume 
that the $E_{n}$ in (12) are Borel sets without changing $\mu(E)$. Then construct $B$ by countable unions and intersections of Borel sets. (iv) follows directly from (11). (v) obtains from the monotonicity of $\mu$ : $\lim \mu\left(E_{n}\right)$ exists and is $\leqslant \mu(E)$. By (iii) we can find Borel sets $B_{n} \supset E_{n}$ with $\mu\left(B_{n}\right)=\mu\left(E_{n}\right)$. But $B_{n} \uparrow B$ and $\mu\left(B_{n}\right) \rightarrow \mu(B)$, so that $\lim \mu\left(E_{n}\right) \geqslant \mu(E)$.

(vi) Let $E$ be $\mu$-measurable and choose a Borel set $B \supset E$ with $\mu(B)=\mu(E)$. Since $\mu(B \backslash E)=0$, we can find a Borel set $B_{1} \supset B \backslash E$ with $\mu\left(B_{1}\right)=0$. Now $B_{2}=B \backslash B_{1}$ is Borel, $B_{2} \subset E$, and $\mu\left(B_{2}\right)=\mu(E)$. The set function $\mu_{1}(F)=\mu\left(F \cap B_{2}\right), F$ Borel, defines a finite Borel measure in $\mathbf{R}^{d} . \mu_{1}$ is therefore inner regular, so there exists a closed set $F \subset B_{2}$ with $\mu_{1}(F)>\mu_{1}\left(B_{2}\right)-\varepsilon$. This gives $\mu(F)>\mu(E)-\varepsilon$.

(vii) We denote the right side of (13) by $\mu^{*}(E)$. (iv) and (v) imply that $\mu(E)=$ $\lim \mu\left(E_{n}\right) \leqslant \lim \tau\left(E_{n}\right)$ for all $E_{n} \uparrow E, E_{n} \in \mathscr{B}\left(\mathbf{R}^{d}\right)$. This gives $\mu(E) \leqslant \mu^{*}(E)$. Conversely, given $\varepsilon>0$ we can cover $E$ by sets $F_{n} \in \mathscr{B}\left(\mathbf{R}^{d}\right)$ with $\sum_{n=1}^{\infty} \tau\left(F_{n}\right)<\mu(E)+\varepsilon$. Take $E_{n}=E \cap\left(\bigcup_{i=1}^{n} F_{i}\right)$. By Lemma 3.1(i) and (ii),

$$
\tau\left(E_{n}\right) \leqslant \sum_{i=1}^{n} \tau\left(F_{i}\right)<\mu(E)+\varepsilon,
$$

so

$$
\mu^{*}(E) \leqslant \mu(E)+\varepsilon .
$$

Notation 5.2. The outer measures generated by the premeasures $\phi-P, \phi-P^{*}$, $\phi-P^{* *}, \phi-Q$ will be denoted by $\phi-p, \phi-p^{*}, \phi-p^{* *}, \phi-q$, respectively.

It follows from Lemma 3.1 that countable sets have zero measure and from Lemma 3.4 that

$$
\phi-p \asymp \phi-p^{* *} \preccurlyeq \phi-p^{*}, \quad \phi-p \preccurlyeq \phi-q .
$$

Let us prove a result which applies to $\phi-p$ and $\phi-q$ by Lemma 3.2.

Lemma 5.3. Suppose $\tau: \mathscr{B}\left(\mathbf{R}^{d}\right) \rightarrow \mathbf{R}^{+}$is monotone subadditive and satisfies $\tau(\bar{A})=$ $\tau(A)$ for all $A$, and let $\mu$ be defined by (13). If $E$ is a compact set such that $\tau(E \cap V)=+\infty$ for all $V$ which are open and meet $E$, then $\mu(E)=+\infty$.

Proof. Suppose $E_{n} \uparrow E$. Since $E$ is closed we must have $E_{N}$ somewhere dense in $E$ (Baire's Theorem) for some $N$. Then take an open set $V$ such that $\overline{E_{N} \cap V}=\overline{E \cap V}$ $\neq \varnothing$ to get

$$
\tau\left(E_{N}\right) \geqslant \tau\left(E_{N} \cap V\right)=\tau \overline{\left(E_{n} \cap V\right)}=\tau \overline{(E \cap V)}=\tau(E \cap V)=+\infty
$$

Using (13) gives $\mu(E)=+\infty$.

We now state the main density theorem which has a similar structure to Theorem 2.1 for Hausdorff measure.

THEOREM 5.4. Suppose $\mu$ is a Borel measure in $\mathbf{R}^{d}$ with $0<\|\mu\|=\mu\left(\mathbf{R}^{d}\right)<+\infty$. $u_{n}(x)$ and $v_{n}(x)$ denote the dyadic and semidyadic cubes, respectively, containing $x$. 
For each $\phi \in \Phi$ there are finite constants $\lambda_{i}>0$ such that, for all $E \subset \mathbf{R}^{d}$,

$$
\begin{aligned}
\lambda_{1} \mu(E) \inf _{x \in E}\left\{\limsup _{r \downarrow 0} \frac{\phi(2 r)}{\mu\left(B_{r}(x)\right)}\right\} & \leqslant \phi-p(E) \\
& \leqslant\|\mu\| \sup _{x \in E}\left\{\limsup _{r \downarrow 0} \frac{\phi(2 r)}{\mu\left(B_{r}(x)\right)}\right\}, \\
\lambda_{2} \mu(E) \inf _{x \in E}\left\{\limsup _{n \rightarrow \infty} \frac{\phi\left(d^{1 / 2} 2^{-n}\right)}{\mu\left(u_{n}(x)\right)}\right\} & \leqslant \phi-p^{*}(E) \\
\lambda_{3} \mu(E) \inf _{x \in E}\left\{\limsup _{n \rightarrow \infty} \frac{\phi\left(d^{1 / 2} 2^{-n}\right)}{\mu\left(v_{n}(x)\right)}\right\} & \leqslant \phi-p^{* *}(E) \sup _{x \in E}\left\{\limsup _{n \rightarrow \infty} \frac{\phi\left(d^{1 / 2} 2^{-n}\right)}{\mu\left(u_{n}(x)\right)}\right\}, \\
& \leqslant\|\mu\| \sup _{x \in E}\left\{\limsup _{n \rightarrow \infty} \frac{\phi\left(d^{1 / 2} 2^{-n}\right)}{\mu\left(v_{n}(x)\right)}\right\} .
\end{aligned}
$$

Proof. Each of the above results can be proved by similar arguments. We give the details for $\phi-p(E)$ which is the hardest case. The right inequality is trivial unless there is a finite $K$ such that, for all $x \in E$,

$$
\limsup _{r \downarrow 0} \frac{\phi(2 r)}{\mu\left(B_{r}(x)\right)}<K .
$$

In this case put $E_{n}=\left\{x \in E: r<1 / n \Rightarrow \phi(2 r)<K \mu\left(B_{r}(x)\right)\right\}$. Since $E_{n} \uparrow E$, $\phi-p(E) \leqslant \sup \phi-p\left(E_{n}\right)$. But if $\mathscr{R}$ is a disjoint family of open balls centred in $E_{n}$ with $\|\mathscr{R}\|<1 / n$, we get $\phi(\mathscr{R})<K\|\mu\|$, so $\phi-p\left(E_{n}\right) \leqslant K\|\mu\|$. Hence, $\phi-p(E) \leqslant$ $K\|\mu\|$ for every such $K$, and the right inequality is established.

Now assume there exists $K>0$ such that, for all $x \in E$,

$$
\limsup _{r \rightarrow 0} \frac{\phi(2 r)}{\mu\left(B_{r}(x)\right)}>K \text {. }
$$

For the left inequality it suffices to prove that

$$
\lambda_{1} K \mu(E) \leqslant \phi-P(E)
$$

(then use (13) and the $\sigma$-additivity of $\mu$ ). We assume $\phi-P(E)<+\infty$. For all $\varepsilon>0$ there exists $\delta$ such that for each packing $\mathscr{R}$ of $E$ by centered balls, $\|\mathscr{R}\| \leqslant \delta$, we have $\phi(\mathscr{R}) \leqslant \phi-P(E)+\varepsilon$. For any ball $B$ denote by $B^{*}$ the ball with the same center and with diameter 5 diam $B$. The family of all balls $B$ centered in $E$ and verifying the inequality $K \mu\left(B^{*}\right) \leqslant \phi\left(\operatorname{diam} B^{*}\right)$ is a Vitali covering of $E$ : a standard argument allows us to select a packing $\mathscr{R}=\left\{B_{i}\right\}$ of $E,\|\mathscr{R}\| \leqslant \delta$, such that for all $n$, $E \subset \cup_{1}^{\infty} B_{i}^{*}$. Therefore,

$$
\begin{aligned}
\mu(E) & \leqslant \sum_{1}^{\infty} \mu\left(B_{i}^{*}\right) \leqslant K^{-1} \sum \phi\left(\operatorname{diam} B_{i}^{*}\right) \\
& \leqslant c_{0}^{3} K^{-1} \phi(\mathscr{R}) \quad \text { from }(1) \\
& \leqslant c_{0}^{3} K^{-1}(\phi-P(E)+\varepsilon) .
\end{aligned}
$$

This proves (15) with $\lambda_{1}=c_{0}^{3}$. 
REMARK 5.5. It is clear from the argument used that if the lim sup is attained uniformly over $E$, the right inequality is valid for $\phi-P$ as well as $\phi-p$.

EXAMPLE 5.6. Consider the set $E_{0}$ introduced in Example 3.5. We take the uniform measure $\mu$ defined on $E_{0}$ by giving to each $u \in \mathscr{C}_{1}$ the mass $|u|^{1 / 2}$. Every interval $u$ verifies

$$
\mu(u) \leqslant|u|^{1 / 2}
$$

so that, for $\phi(s)=s^{1 / 2}$, Theorem 5.4 implies $\phi-p\left(E_{0}\right)>0$. For an upper bound we already know that $\phi-p\left(E_{0}\right) \leqslant \phi-P\left(E_{0}\right)<+\infty$.

Taking away the countable set of endpoints of the complementary intervals, we get $E_{1}$ such that

$$
0<\phi-p^{*}\left(E_{0}\right)=\phi-p^{*}\left(E_{1}\right) \leqslant \phi-P^{*}\left(E_{1}\right)<+\infty .
$$

Now let us consider $\phi-q$ : the uniform structure of $E_{0}$ tells us that for all $V$ open, $V \cap E_{0} \neq \varnothing$, we have

$$
\phi-Q\left(E_{0} \cap V\right) \geqslant \phi-P^{*}\left(E_{0} \cap V\right)=+\infty .
$$

This shows that $\phi-q$ and $\phi-p^{*}$ are not equivalent and the conclusion of Lemma 5.3 does not hold for $\phi-p^{*}$.

EXAMPLE 5.7. Clearly, $\phi-p$ is invariant for translations. However, the following argument shows that, for the set $E_{0}$ of Examples 3.5 and 5.6 and $\phi(s)=s^{1 / 2}$, we can find a real number $\alpha$ such that

$$
\phi-p^{*}\left(E_{0}+\alpha\right)=+\infty .
$$

This shows that $\phi-p$ and $\phi-p^{*}$ are not equivalent and, moreover, $\phi-p^{*}$ is not invariant for translations even in a weak sense.

$\mu$ is the uniform measure on $E_{0}$ defined above. We choose $\alpha=\sum_{i=1}^{\infty} 2^{-2^{i}}$. Let us fix an integer $k \geqslant 1$. Let $x=\sum_{1}^{\infty} a_{n}(x) 4^{-n}, a_{n}(x)=0$ or 3 , be a typical element of $E_{0}$. The event

$$
A_{n}(x): a_{2^{n}-k+1}(x)=\cdots=a_{2^{n}}=3
$$

occurs infinitely often for $\mu$-almost all $x$ : this can be proved by a Borel-Cantelli argument. Now put $y=x+\alpha, b_{n}$ the right endpoint of $u_{n}(x), b_{n}^{\prime}$ the left endpoint of $u_{n}(y)$. We want to show that, provided $A_{n}(x)$, the distance between $b_{2^{n}-k}^{\prime}$ and $b_{2^{n}-k}+\alpha$ is small, which gives a small measure to $u_{2^{n}-k}(y)$.

If $A_{n}(x)$ is true, then $0<b_{2^{n}-k}-x \leqslant 2^{-2^{n}}$, so that $0<b_{2^{n}-k}+\alpha-y \leqslant 2^{-2^{n}}$. But $b_{2^{n}-k}+\alpha$ can be written $z \cdot 2^{-2^{n}+k}+\eta$, where $z \in \mathbf{Z}$ and $\eta=\sum_{n}^{\infty} 2^{-2^{i}}<2^{-2^{n}+1}$. Therefore $b_{2^{n}-k}^{\prime}=z \cdot 2^{-2^{n}+k}$ and $0<b_{2^{n}-k}+\alpha-b_{2^{n}-k}^{\prime}<2^{-2^{n}+1}$. Let us take for measure $\mu^{*}$ on $E_{0}+\alpha$ the translate of $\mu$. This last inequality implies

$$
\mu^{*}\left(u_{2^{n}-k}(y)\right)=\mu^{*}\left(\left[b_{2^{n}-k}^{\prime}, b_{2^{n}-k}+\alpha[),\right.\right.
$$

so that, using (16) for $\mu^{*}$,

$$
\mu^{*}\left(u_{2^{n}-k}(y)\right) \leqslant 2^{\left(-2^{n}+1\right) / 2} .
$$

This gives

$$
\phi\left(\left|u_{2^{n}-k}(y)\right|\right) / \mu^{*}\left(u_{2^{n}-k}(y)\right) \geqslant 2^{(k-1) / 2}
$$


infinitely often for $\mu^{*}$-almost all $y$ in $E_{0}+\alpha$. Theorem 5.4 implies that

$$
\phi-p^{*}\left(E_{0}+\alpha\right) \geqslant \lambda_{2} 2^{(k-1) / 2},
$$

where $k$ is arbitrarily large, hence (17) holds.

EXAMPLE 5.8. It is easy to check that the triadic Cantor set $C \subset[0,1]$ satisfies

$$
0<e^{\alpha}-p(C)<+\infty \text { for } \alpha=\log 2 / \log 3 \text {. }
$$

CONJECTURE. This set $C$ is such that $e^{\alpha}-p^{*}(C)=+\infty$. In order to prove this, one seems to need a strong independence result concerning the expansions of a real to bases 2 and 3.

COROLlaRy 5.9. Each of the outer measures $\phi-p, \phi-p^{*}, \phi-q$ defines the same dimensional index.

Proof. This follows from Corollary 3.9. This index is denoted Dim or $\hat{\Delta}-$ see Tricot [19].

Since $\phi-m \preccurlyeq \phi-p \preccurlyeq \phi-P$, we have

$$
\operatorname{dim} \leqslant \operatorname{Dim} \leqslant \Delta .
$$

We now have to make a choice for "packing measure". We discard $\phi-q$ because there are no infinite sets of finite positive measure in $\mathbf{R}$. The above examples show that $\phi-p^{*}$ is not translation invariant. We could take either $\phi-p$ or $\phi-p^{* *}$, as both satisfy the density theorem and are equivalent. However, $\phi-p^{* *}$ involves the use of a particular net, and it is unlikely to be invariant for translations so we make

Definition 5.10. Given $\phi \in \Phi$, the outer measure $\phi-p$ is called the $\phi$-packing measure.

We now compare $\phi-p$ with the Hausdorff measure $\phi-m$ :

LEMMA 5.11. For all $E \subset \mathbf{R}^{d}, \phi-m(E) \leqslant \phi-p(E)$.

Proof. Let us assume first that $E$ is a bounded, Borel set. It is enough to prove that

$$
\phi-m(E) \leqslant \phi-P(E) .
$$

Assume $\phi-P(E)<+\infty$. Let $\varepsilon>0$ and $\rho_{1}=\rho_{1}(E, \varepsilon)$ be small enough to ensure that for each family $\left(U_{i}\right)$ of Borel sets with $\operatorname{diam} U_{i} \leqslant \rho_{1}$, we have

$$
\phi-m\left(E \cap\left(\cup U_{i}\right)\right) \leqslant \sum \phi\left(\operatorname{diam} U_{i}\right)+\varepsilon
$$

(see $[1, \S 7])$. Let $\rho_{2}$ be small enough to ensure that for each packing of $E$ by open balls $B_{i}$ of diameter $\leqslant \rho_{2}$ centered in $E$ we have

$$
\sum \phi\left(\operatorname{diam} B_{i}\right) \leqslant \phi-P(E)+\varepsilon .
$$

By a classical Vitali argument we can choose a packing $\left(B_{i}\right)$ of such balls, with $\operatorname{diam} B_{i} \leqslant \min \left(\rho_{1} / 5, \rho_{2}\right)$, and such that for all $n, E-\cup_{1}^{n} B_{i} \subset \cup_{n+1}^{\infty} B_{i}^{*}$, where $B_{i}^{*}$ is the ball with the same center as $B_{i}$ and with radius multiplied by a factor of 5 . Therefore,

$$
\phi-m\left(E-\bigcup_{1}^{n} B_{i}\right) \leqslant \phi-m\left(E \cap\left(\bigcup_{n+1}^{\infty} B_{i}^{*}\right)\right),
$$


and given $\operatorname{diam} B_{i}^{*} \leqslant \rho_{1}$,

$$
\phi-m\left(E-\bigcup_{1}^{n} B_{i}\right) \leqslant \sum_{n+1}^{\infty} \phi\left(\operatorname{diam} B_{i}^{*}\right)+\varepsilon \leqslant c_{0}^{3} \sum_{n+1}^{\infty} \phi\left(\operatorname{diam} B_{i}\right)+\varepsilon,
$$

where $\sum_{n+1}^{\infty} \phi\left(\operatorname{diam} B_{i}\right)$ tends to 0 as $n \rightarrow \infty$ since the series converges. Therefore,

$$
\begin{aligned}
\phi-m(E) & \leqslant \phi-m\left(E \cap\left(\bigcup_{1}^{\infty} B_{i}\right)\right)+\varepsilon \\
& \leqslant \sum_{1}^{\infty} \phi\left(\operatorname{diam} B_{i}\right)+2 \varepsilon \leqslant \phi-P(E)+3 \varepsilon .
\end{aligned}
$$

This proves (19) for bounded, Borel sets. But both measures are Borel regular so the result follows for any bounded subset of $\mathbf{R}^{d}$. The extension of Lemma 5.11 to unbounded sets is immediate.

REMARK 5.12. It will usually be the case that $\phi-m(E)<\phi-p(E)$ unless they are both 0 or $+\infty$. The condition $0<\phi-m(E)=\phi-p(E)<+\infty$ is a very strong regularity condition which will be studied in a subsequent paper [16].

6. Sojourn time for Brownian motion. Before we can attack the problem of packing measure for sample paths we need precise information about the small tail of the distribution of the total time spent in a ball. We restrict attention to Brownian motion in $\mathbf{R}^{d}(d \geqslant 3)$ where the process is transient and, therefore, the total sojourn time is finite. The case $d=1$ is trivial, and a solution for $d=2$ will require some additional techniques.

$X_{t}=X_{t}(\omega),-\infty<t<+\infty$, is a standard version of the Wiener process in $\mathbf{R}^{d}$. $P=P^{0}$ is the Wiener measure for $X_{0}=0$. We need several associated random variables. Let

$$
M_{d}=\sup \left\{\left\|X_{t}-X_{0}\right\|, 0 \leqslant t \leqslant 1\right\} .
$$

For $r>0, P_{d}(r)$ denotes the first passage time out of the ball $B_{r}(0)$, given $X_{0}=0$ :

$$
P_{d}(r)=\inf \left\{t>0: X_{t} \notin B_{r}(0)\right\} .
$$

The total time spent in $B_{r}(0)$ after 0 , given $X_{0}=0$, is

$$
U_{d}(r)=\left|\left\{t>0: X_{t} \in B_{r}(0)\right\}\right|,
$$

while the corresponding time before 0 is

$$
V_{d}(r)=\left|\left\{t<0: X_{t} \in B_{r}(0)\right\}\right|,
$$

and the sojourn time in $B_{r}(0)$, given $X_{0}=0$, is

$$
T_{d}(r)=U_{d}(r)+V_{d}(r)
$$

We are interested in the small values of $T_{d}(r)$ as $r \downarrow 0 . U_{d}(r)$ and $V_{d}(r)$ are independent positive random variables with the same distribution, so $T_{d}$ is small only if $U_{d}$ and $V_{d}$ are small. It is for this reason that our argument is more complicated than that in [2], for there the large values of $T_{d}$ were relevant and these are the same size as the large values of $U_{d}$ or $V_{d}$.

The trajectory of the process on $[0,1]$ is denoted

$$
E(\omega)=\left\{x \in \mathbf{R}^{d}: x=X_{t} \text { for some } t \in[0,1]\right\} .
$$


Since $X_{t}$ is continuous a.s. $E(\omega)$ is a compact set. For $n \in \mathbf{N}$ we put

$N_{n}(\omega)=$ number of dyadic cubes of side $2^{-n}$ containing a point of $E(\omega)$.

We can now state some elementary results.

LEMMA 6.1. Suppose $\|x\|>r$; then

$$
P\left\{B_{r}(x) \text { is hit by } X_{t} \text { for } t>0\right\}=(r /\|x\|)^{d-2} .
$$

This is well known: it follows from the fact that the hitting probability of a ball starting from $y$ is a harmonic function of $y$ with boundary value 1 on the surface of $B_{r}(x)$.

LEMMA 6.2. There are positive constants $c_{1}, c_{2}$ such that, for $r>1$,

$$
c_{1} r^{d-2} e^{-r^{2} / 2} \leqslant P\left\{M_{d} \geqslant r\right\} \leqslant c_{2} r^{d-2} e^{-r^{2} / 2} .
$$

Proof. The reflection principle gives

$$
P\left\{\left\|X_{1}\right\| \geqslant r\right\} \leqslant P\left\{M_{d} \geqslant r\right\} \leqslant 2 P\left\{\left\|X_{1}\right\| \geqslant r\right\} .
$$

The result now follows using the large tail of the normal distribution.

LEMMA 6.3. There is a positive constant $c_{3}$ such that

$$
E\left(N_{n}(\omega)\right)<c_{3} 2^{2 n} \text { for all } n \in \mathbf{N} \text {. }
$$

This can be deduced from Lemmas 6.1 and 6.2 by a standard calculation, or we can obtain it as a corollary of the results in [2].

LEMMA 6.4. For $0<t<1$,

$$
c_{1} t^{2-d / 2} e^{-1 / 2 t} \leqslant P\left\{U_{d}(1) \leqslant t\right\} \leqslant c_{2} t^{2-d / 2} e^{-1 / 2 t} .
$$

Proof. It was proved in [2] that $U_{d}(1)$ has the same distribution as $P_{d-2}(1)$. Hence,

$$
P\left\{U_{d}(1) \leqslant t\right\}=P\left\{P_{d-2}(1) \leqslant t\right\}=P\left\{P_{d-2}\left(t^{-1 / 2}\right) \leqslant 1\right\}=P\left\{M_{d-2} \geqslant t^{-1 / 2}\right\},
$$

using the scaling property for Brownian motion for the third term. The result now follows from Lemma 6.2.

LEMMA 6.5. For each $\varepsilon>0$ there is a $t_{1}=t_{1}(\varepsilon)>0$ such that

$$
e^{-(2+\varepsilon) / t} \leqslant P\left\{T_{d}(1) \leqslant t\right\} \leqslant e^{-(2-\varepsilon) / t} \text { for } 0<t<t_{1} .
$$

Proof. Since $U_{d}(1)$ and $V_{d}(1)$ are independent with the same distribution, we get

$$
\begin{aligned}
P\left\{T_{d}(1) \leqslant t\right\} & \geqslant\left[P\left\{U_{d}(1) \leqslant \frac{1}{2} t\right\}\right]^{2} \\
& \geqslant K t^{-d+4} e^{-2 / t} \quad \text { by Lemma } 6.4 \\
& \geqslant e^{-(2+\varepsilon) / t} \quad \text { if } t \text { is small enough. }
\end{aligned}
$$

A fairly crude method gives the right inequality. Put $G(s)=P\left\{U_{d}(1) \leqslant s\right\}$ and fix an integer $k$. Then

$$
\begin{aligned}
P\left\{T_{d}(1) \leqslant t\right\} & =\int_{0}^{t} G(t-s) d G(s)=\sum_{i=1}^{k} \int_{(i-1) t / k}^{i t / k} G(t-s) d G(s) \\
& \leqslant \sum_{i=1}^{k} G\left(\frac{k-i+1}{k} t\right) G\left(\frac{i}{k} t\right) .
\end{aligned}
$$


Now use Lemma 6.4 with distinct arguments for $d \geqslant 4$ and $d=3$ to give

$$
G\left(\frac{k-i+1}{k} t\right) G\left(\frac{i}{k} t\right) \leqslant C(k) t^{4-d} \exp \left[-\frac{1}{2 t} \frac{k^{2}+k}{i(k-i+1)}\right],
$$

where $C(k)=\mathcal{O}\left(k^{d-3}\right)$ as $k \rightarrow \infty$. But, for $1 \leqslant i \leqslant k,\left(k^{2}+k\right) / i(k-i+1) \geqslant$ $4(1-1 / k)$, so

$$
P\left\{T_{d}(1) \leqslant t\right\} \leqslant k C(k) t^{4-d} \exp [-(2 / t)(1-1 / k)] .
$$

Given $\varepsilon>0$ choose $k>3 / \varepsilon$ and $t$ small enough to give

$$
P\left\{T_{d}(1) \leqslant t\right\} \leqslant e^{-(2-\varepsilon) / t} \text { for } 0<t<t_{1} .
$$

REMARK 6.6. It is clear that we could tighten up the above argument considerably but not enough to give an exact asymptotic value for $P\left\{T_{d}(1) \leqslant t\right\}$ as $t \downarrow 0$. If we could have proved the following, it would have simplified our task in the next section.

CONJECTURE 6.7. There are constants $K=K(d), \lambda=\lambda(d)$, such that

$$
P\left\{T_{d}(1) \leqslant t\right\} \sim K t^{\lambda} e^{-2 / t} \text { as } t \downarrow 0 .
$$

It seems likely that this should follow from a general result on the small tail of the distribution of the sum of two positive independent random variables, but the existing techniques are not good enough.

THEOREM 6.8. Suppose $T_{d}(r)$ is the total time spent in the ball $B_{r}(0)$ by a Brownian motion process in $\mathbf{R}^{d}(d \geqslant 3), X_{t},-\infty<t<+\infty$, with $X_{0}=0$. Then

$$
\liminf _{r \downarrow 0} \frac{T_{d}(r)}{\psi(r)}=2 \text { a.s., }
$$

where $\psi(r)=r^{2} / \log |\log r|$.

Proof. We fix $d$ throughout the proof and omit it. In the easier direction a standard Borel-Cantelli argument will work. Fix $\gamma<2$. For $k=2,3, \ldots$ let $a_{k}=$ $\exp (-k / \log k), E_{k}=\left\{\omega: T\left(a_{k}\right) \leqslant \gamma \psi\left(a_{k}\right)\right\}$. Let $0<\varepsilon<1$. Using the scaling property,

$$
\begin{aligned}
P\left(E_{k}\right) & =P\left\{T(1) \leqslant \gamma / \log \left|\log a_{k}\right|\right\} \leqslant P\{T(1) \leqslant \gamma /(1-\varepsilon) \log k\} \\
& \leqslant k^{-\alpha}, \quad \text { where } \alpha=(1-\varepsilon)(2-\varepsilon) / \gamma
\end{aligned}
$$

by Lemma 6.5 , when $k$ is large enough. Taking $\varepsilon$ small enough to make $\alpha>1$, we have $\sum P\left(E_{k}\right)$ converges, so, with probability 1 , there exists $k_{1}=k_{1}(\omega)$ for which $k \geqslant k_{1} \Rightarrow T\left(a_{k}\right)>\gamma \psi\left(a_{k}\right)$. Thus

$$
\liminf _{k \rightarrow \infty} \frac{T\left(a_{k}\right)}{\psi\left(a_{k}\right)} \geqslant \gamma \text { a.s. }
$$

But $T_{d}(r)$ and $\psi(r)$ are monotone for small $r$ and

$$
\lim _{k \rightarrow \infty} \frac{\psi\left(a_{k}\right)}{\psi\left(a_{k+1}\right)}=1 \text {. }
$$

Hence,

$$
\underset{r \downarrow 0}{\liminf _{r}} \frac{T(r)}{\psi(r)} \geqslant \gamma \quad \text { a.s. }
$$


Since $\gamma$ can be chosen arbitrarily close to 2 , we get

$$
\underset{r \downarrow 0}{\liminf } \frac{T(r)}{\psi(r)} \geqslant 2 \text { a.s. }
$$

In the other direction we require precise estimates of probabilities, so we have to use the fact that $T(r)$ is the sum of two independent random variables $T(r)=U(r)+$ $V(r)$. We now fix $\gamma>2$ and show that, for $b_{k}=\exp \left(-k(\log k)^{2}\right), k=2,3, \ldots$, infinitely many of the events

$$
D_{k}=\left\{\omega: T\left(a_{k}\right) \leqslant \gamma \psi\left(a_{k}\right)\right\}
$$

occur with probability 1 . This is enough to give

$$
\underset{r \downarrow 0}{\liminf } \frac{T(r)}{\psi(r)} \leqslant 2 \quad \text { a.s., }
$$

which with (20) will complete the proof of the theorem. The first step uses the minimum 'rate of escape' to show that there is no contribution to $T\left(b_{k}\right)$ from values of $t$ with $|t|$ much greater than $b_{k}^{2}$. To be precise put $\tau=k^{3} b_{k}^{2}$ and

$$
T\left(b_{k}, \tau_{k}\right)=\mid\left\{t \in \mathbf{R}:|t| \leqslant \tau_{k} \text { and }\left\|X_{t}\right\| \leqslant b_{k}\right\} \mid \text {. }
$$

Then the event $T\left(b_{k}, \tau_{k}\right) \neq T\left(b_{k}\right)$ is contained in

$$
\left\{\omega: t>\tau_{k} \text { with }\left\|X_{t}\right\| \leqslant b_{k}\right\} \cup\left\{\omega: t<-\tau_{k} \text { with }\left\|X_{t}\right\| \leqslant b_{k}\right\} .
$$

The estimates for delayed hitting probability in [3] give

$$
P\left\{T\left(b_{k}, \tau_{k}\right) \neq T\left(b_{k}\right)\right\}=\mathcal{O}\left(k^{-3 / 2}\right),
$$

so that a.s. there exists $k_{2}=k_{2}(\omega)$ for which

$$
k \geqslant k_{2} \Rightarrow T\left(b_{k}\right)=T\left(b_{k}, \tau_{k}\right) \text {. }
$$

Using the large tail of the normal distribution to estimate $P\left\{\left\|X_{\tau_{k}}\right\|>k^{2} b_{k}\right\}$, we can show that a.s. there exists $k_{3}=k_{3}(\omega)$ for which

$$
k \geqslant k_{3} \Rightarrow\left\|X_{\tau_{k}}\right\| \leqslant k^{2} b_{k} \text { and }\left\|X_{-\tau_{k}}\right\| \leqslant k^{2} b_{k} .
$$

Now put $e_{k}=b_{k}+(k+1)^{2} b_{k+1}$ and note that

$$
k \geqslant k_{3} \Rightarrow B_{b_{k}}(0) \subset B_{e_{k}}\left(X_{\tau_{k+1}}\right) \text {. }
$$

Define new sections of sojourn time

$$
\begin{aligned}
& U\left(t_{0}, r, \tau\right)=\mid\left\{t: t_{0} \leqslant t \leqslant t_{0}+\tau \text { and }\left\|X_{t}-X_{t_{0}}\right\| \leqslant r\right\} \mid, \\
& V\left(t_{0}, r, \tau\right)=\mid\left\{t: t_{0}-\tau \leqslant t \leqslant t_{0} \text { and }\left\|X_{t}-X_{t_{0}}\right\| \leqslant r\right\} \mid .
\end{aligned}
$$

By (24), for $k \geqslant k_{3}$,

$$
U\left(0, b_{k}, \tau_{k}\right) \leqslant \tau_{k+1}+U\left(t_{k+1}, e_{k}, \tau_{k}-\tau_{k+1}\right) .
$$

But now the events

$$
A_{k}=\left\{\omega: U\left(\tau_{k+1}, e_{k}, \tau_{k}-\tau_{k+1}\right) \leqslant \frac{1}{2} \gamma \psi\left(b_{k}\right)-\tau_{k+1}\right\}
$$

are independent, and

$$
P\left(A_{k}\right) \geqslant P\left\{U\left(e_{k}\right) \leqslant \frac{1}{2} \gamma \psi\left(b_{k}\right)-\tau_{k+1}\right\}=P\left\{U(1) \leqslant y_{k}\right\},
$$


where

$$
y_{k}=\left(\frac{1}{2} \gamma \psi\left(b_{k}\right)-\tau_{k+1}\right) / e_{k}^{2} \sim \frac{1}{2} \gamma / \log k \quad \text { as } k \rightarrow \infty .
$$

It follows from Lemma 6.4 that, for each $\varepsilon>0$, whenever $k$ is large enough,

$$
P\left(A_{k}\right) \geqslant k^{-(1+\varepsilon) / \gamma} \text {. }
$$

The same result holds for the symmetrical event

$$
B_{k}=\left\{\omega: V\left(-\tau_{k+1}, e_{k}, \tau_{k}-\tau_{k+1}\right) \leqslant \frac{1}{2} \gamma \psi\left(b_{k}\right)-\tau_{k+1}\right\} .
$$

The events $A_{k}, B_{k}$ are independent, so we get

$$
P\left(A_{k} \cap B_{k}\right) \geqslant k^{-2(1+\varepsilon) / \gamma} .
$$

But for $k=2,3, \ldots$, the events $A_{k} \cap B_{k}$ depend on disjoint time intervals and are, therefore, independent. It follows that, with probability 1 , for each $\gamma>2, A_{k} \cap B_{k}$ happens infinitely often. We now combine (22) and (25) to deduce that $D_{k}$ defined by (21) happens infinitely often a.s.

REMARK 6.9. There are two differences between the result of Theorem 6.8 and the corresponding lim sup law obtained in [2]. Firstly, the asymptotic value does not depend on $d \geqslant 3$. Secondly, the constant is different for a one-sided law. A simplified version of the above argument shows that

$$
\liminf _{r \rightarrow 0} \frac{U_{d}(r)}{\psi(r)}=\frac{1}{2} \quad \text { a.s. }
$$

Corollary 6.10. For fixed $t_{0}$ let $T_{d}\left(t_{0}, r\right)$ denote the total time spent in $B_{r}\left(X_{t_{0}}\right)$ by a Brownian motion process in $\mathbf{R}^{d}(d \geqslant 3)$. Then

$$
\liminf _{r \downarrow 0} \frac{T_{d}\left(t_{0}, r\right)}{\psi(r)}=2 \text { a.s. }
$$

Proof. $Y_{s}=X_{t_{0}+s}-X_{t_{0}},-\infty<s<\infty$, defines a version of the Brownian motion process for which $Y_{0}=0$. Apply the theorem to $Y$.

7. The exact packing measure of Brownian motion. Our object in this section is to prove that

$$
\psi(r)=r^{2} / \log |\log r|
$$

is the correct function in $\Phi$ to give a finite positive $\psi$-packing measure for a piece of Brownian trajectory in $\mathbf{R}^{d}(d \geqslant 3)$. To obtain an upper bound we use a result which may be more general than we need.

LEMMA 7.1. Suppose $Y_{t}(\omega)$ is any standard process in $\mathbf{R}^{d}(d \geqslant 1)$ and $E(\omega)=\{x$ : $x=Y_{t}$ for some $\left.t \in[0,1]\right\}$. Let $N_{n}(\omega)$ be the number of dyadic cubes of side $2^{-n}$ which meet $E(\omega), \mu=\mu(\omega)$ be a finite Borel measure in $\mathbf{R}$, and write, for $\lambda>0$,

$$
q_{n, \lambda}=\sup _{x \in E(\omega)} P\left\{\mu\left(B_{2^{-n}}(x)\right)<\lambda \phi\left(2^{-n}\right)\right\} .
$$

If $\sum_{n=1}^{\infty}\left[q_{n, \lambda} \phi\left(2^{-n}\right) E\left(N_{n}(\omega)\right)\right]^{1 / 2}<+\infty$ for some $\lambda>0$, then $\phi-P(E(\omega))<+\infty$ a.s. 
Proof. Using (1) and the trivial inequality $N_{n} \leqslant N_{n+2}$, we see that the hypothesis of Lemma 7.1 implies

$$
\sum\left[q_{n+2, \lambda} \phi\left(2^{-n}\right) E\left(N_{n}(\omega)\right)\right]^{1 / 2}<+\infty .
$$

A semidyadic cube $v$ of side $2^{-n}$ is called 'good' if $\mu(v) \geqslant \lambda \phi\left(2^{-n-2}\right)$; otherwise it is 'bad'. If $v=v_{n}(x)$ then $v$ contains $B_{2^{-n-2}}(x)$ so that, for $x \in E(\omega)$,

$$
P\left(v_{n}(x) \text { is bad }\right) \leqslant q_{n+2, \lambda} .
$$

There are less than $K N_{n}$ such cubes $v_{n}(x)(K=K(d))$, so if $M_{n}$ is the number of bad cubes,

$$
E\left(M_{n}(\omega)\right)<K q_{n+2, \lambda} E\left(N_{n}(\omega)\right)
$$

Hence,

$$
P\left\{M_{n}>\left[q_{n+2, \lambda} E\left(N_{n}\right) / \phi\left(2^{-n}\right)\right]^{1 / 2}\right\} \leqslant K\left[q_{n+2, \lambda} \phi\left(2^{-n}\right) E\left(N_{n}\right)\right]^{1 / 2} .
$$

Using Borel-Cantelli and (26) a.s., we obtain that there is $n_{1}=n_{1}(\omega)$ such that

$$
M_{n} \leqslant\left[q_{n+2, \lambda} E\left(N_{n}\right) / \phi\left(2^{-n}\right)\right]^{1 / 2} \text { for } n \geqslant n_{1} \text {. }
$$

Now if $\mathscr{R}$ is a family of disjoint cubes of $\Gamma^{* *}$ with $\|\mathscr{R}\| \leqslant 2^{-N}, N \geqslant n_{1}$, the contribution of the 'bad' cubes to the sum $\phi(\mathscr{R})$ becomes negligible, for

$$
\begin{aligned}
\sum\{\phi(d(v)): v \in \mathscr{R}, v \text { is bad }\} & \leqslant \sum_{n=N}^{\infty} M_{n} \phi\left(d 2^{-n}\right) \\
& \leqslant c \sum_{n=N}^{\infty}\left|q_{n+2, \lambda} \phi\left(2^{-n}\right) E\left(N_{n}\right)\right|^{1 / 2}
\end{aligned}
$$

and this converges to 0 as $N \rightarrow \infty$ by (26). Also

$$
\sum\{\phi(d(V)): v \in \mathscr{R}, v \text { is } \operatorname{good}\} \leqslant c_{0}^{r} \sum \phi\left(\frac{1}{4} \text { side of } v\right) \leqslant \frac{c_{0}^{r}}{\lambda}\|\mu\|,
$$

where $r$ is an integer such that $2^{r-2}>d^{1 / 2}$. Thus, we have proved that $\phi-P^{* *}(E(\omega)) \leqslant c_{1}<+\infty$. By Lemma 3.4 this tells us that there is a $K_{1}$ s.t.

$$
\phi-P(E(\omega)) \leqslant K_{1}<+\infty \text { a.s. }
$$

Corollary 7.2. If $X_{t}$ is a standard Brownian motion in $\mathbf{R}^{d}(d \geqslant 3)$, then $\psi-p(E(\omega)) \leqslant K_{1}<+\infty$, where $\psi(r)=r^{2} / \log |\log r|$.

Proof. We define $\mu=\mu(\omega)$ by the projecting Lebesgue measure from [0,1] onto $E(\omega)$. Thus for Borel $A, \mu(A)=\left|\left\{t \in[0,1]: X_{t} \in A\right\}\right|$. Clearly, $\mu\left(\mathbf{R}^{d}\right)=\mu(E(\omega))=$ 1. Now suppose $0<\lambda<1 / 4$. For $t \in[0,1]$,

$$
\begin{aligned}
P\left\{\mu\left(B_{2^{-n}}\left(X_{t}\right)\right) \leqslant \lambda \psi\left(2^{-n}\right)\right\} & \leqslant P\left\{U\left(2^{-n}\right) \leqslant \lambda \psi\left(2^{-n}\right)\right\} \\
& \leqslant P\left\{U(1) \leqslant \lambda 2^{2 n} \psi\left(2^{-n}\right)\right\} \leqslant P\{U(1) \leqslant \lambda / \log n\} .
\end{aligned}
$$

Using Lemma 6.4 gives

$$
q_{n, \lambda} \leqslant c_{2} \lambda^{2-d / 2}(\log n)^{-2+d / 2} n^{-1 / 2 \lambda} .
$$

Using Lemma 6.3 satisfies the hypothesis of Lemma 7.1 for $\lambda<1 / 4$. Thus we have

$$
\psi-p(E(\omega)) \leqslant \psi-P(E(\omega)) \leqslant K_{1}<+\infty \text { a.s. }
$$


REMARK 7.3. There is an alternative proof of the result $\psi-p(E(\omega)) \leqslant K_{1}$. If we use Theorem 4.1 and the result about strong variation proved in [14], $V_{\psi}(X(I))=2$ a.s., we get an immediate upper bound.

LEMMA 7.4. If $X_{t}$ is a standard Brownian motion in $\mathbf{R}^{d}(d \geqslant 3)$, there is a constant $K_{2}$ such that $\psi-p(E(\omega)) \geqslant K_{2}>0$ a.s.

Proof. We apply the density theorem to the measure $\mu$ defined above. By Corollary 6.10 , for each fixed $t_{0} \in(0,1)$, almost surely

$$
\limsup _{r \downarrow 0} \frac{\psi(r)}{\mu\left(B_{r}\left(X\left(t_{0}\right)\right)\right)}=\frac{1}{2} .
$$

By a Fubini argument the set of $t_{0} \in(0,1)$ for which (27) is true has full measure, so the corresponding $E_{1} \subset E(\omega)$ satisfies $\mu\left(E_{1}\right)=1$. Apply Theorem 5.4 to the set $E_{1}$ to get

$$
\psi-p(E) \geqslant \psi-p\left(E_{1}\right) \geqslant \lambda_{1} / 2>0 \text { a.s. }
$$

We can now state our main result.

TheOREM 7.5. Suppose $X_{t}(-\infty<t<\infty)$ is a standard Brownian motion in $\mathbf{R}^{d}$ $(d \geqslant 3)$. Almost surely there are finite positive constants $\rho_{d}$ such that $\psi-p(X(A))=$ $\rho_{d}|A|$ for every Borel set $A \subset \mathbf{R}$, where

$$
X(A)=\left\{x \in \mathbf{R}^{d}: t \in A \text { with } X_{t}=x\right\} .
$$

Proof. Corollary 7.2 and Lemma 7.3 ensure that

$$
0<K_{2} t \leqslant \psi-p(X(0, t)) \leqslant K_{1} t
$$

for each fixed $t>0$. Let $Y_{t}=\psi-p(X(0, t))$. Let us show that $Y_{t}$ is an independent increment process, that is, for any fixed times $0<t_{1}<t_{2}$, putting $Z=X\left(0, t_{1}\right) \cap$ $X\left(t_{1}, t_{2}\right)$ :

$$
\psi-p(Z)=0 \quad \text { a.s. }
$$

(In fact if $d \geqslant 4, Z$ is empty a.s.; see [4].) From Lemmas 3.4 and 5.1(iv), it suffices to show that $\psi-P^{*}(Z)=0$, or rather the stronger result

$$
\forall \varepsilon>0, \quad e^{1+\varepsilon}-P^{*}(Z)=0 .
$$

Let $D_{n}$ be the number of dyadic cubes of side $2^{-n}$ hit by $Z$, that is, by both $X\left(0, t_{1}\right)$ and $X\left(t_{1}, t_{2}\right)$. A calculation of the first moment of $D_{n}$ and an application of Borel-Cantelli shows that a.s. there exists $n_{0}=n_{0}(\omega, \varepsilon)$ such that $n \geqslant n_{0} \Rightarrow D_{n}<$ $2^{n(1+\varepsilon / 2)}$. For any $N \geqslant n_{0}$ and for any packing $\mathscr{R}$ of $Z$ by dyadic cubes of side $\leqslant 2^{-N}$ containing a point of $Z$, we get

$$
e^{1+\varepsilon}(\mathscr{R}) \leqslant \sum_{N}^{\infty}\left(d^{1 / 2} 2^{-n}\right)^{1+\varepsilon} D_{n}<+\infty .
$$

Letting $N \rightarrow \infty$ it follows that $\phi-P^{*}(Z)=0$ (actually this proves that $\Delta(Z) \leqslant 1$, and since $\operatorname{dim}(Z)=1$ [5], we get $\operatorname{dim}(Z)=\operatorname{Dim}(Z)=\Delta(Z)=1$ ).

(29) being proved, it follows that $Y_{t}$ is a Lévy process. Using (28) and an argument in [15], we deduce that there is a constant $\rho_{d}$ such that

$$
\psi-p(X(0, t))=\rho_{d} \text { for all } t>0 \text { a.s. }
$$


A similar argument works for $\psi-p(X(-t, 0))$. It is now immediate that

$$
\psi-p\left(X\left(t_{1}, t_{2}\right)\right)=\rho_{d}\left(t_{2}-t_{1}\right)
$$

for all rational $t_{1}, t_{2}$ and, therefore, for all $t_{1}, t_{2}$ by monotonicity. The result now follows for any Borel set $A$ since we can approximate $A$ by a union of intervals.

REMARK 7.6. We have seen that (27) and $V_{\psi}\left(X_{t}\right)$ do not depend on the dimension $d \geqslant 3$. It seems likely that the constants $\rho_{d}$ in Theorem 7.5 are the same for each $d \geqslant 3$. It would be interesting to find $\rho_{d}$.

\section{REFERENCES}

1. A. S. Besicovitch, On the fundamental geometrical properties of linearly measurable plane sets of points, Math. Ann. 98 (1928), 422-464.

2. Z. Ciesielski and S. J. Taylor, First passage times and sojourn times for Brownian motion in space and the exact Hausdorff measure of the sample path, Trans. Amer. Math. Soc. 103 (1962), 434-450.

3. A. Dvoretzky and P. Erdös, Some problems on random walk in space, Proc. Second Berkeley Sympos., Univ. of California Press, Berkeley, 1950, pp. 353-367.

4. A. Dvoretzky, P. Erdös and S. Kakutani, Double points of Brownian paths in n-space, Acta Sci. Math. Szeged 12 (1950), 75-81.

5. B. E. Fristedt, An extension of a theorem of S. J. Taylor concerning the multiple points of the symmetric stable process, Z. Warsch. Verw Gebiete 9 (1967), 62-64.

6. O. Frostman, Potentiel d'équilibre et capacité des ensembles, Medd Lunds Univ. Mat. Semin. 3 (1935).

7. C. Goffman and J. J. Loughlin, Strong and weak $\phi$-variation of Brownian motion, Indiana Univ. Math. J. 22 (1972), 135-138.

8. M. Munroe, Measure and integration, Addison-Wesley, 1971.

9. C. A. Rogers, Hausdorff measures, Cambridge Univ. Press, 1970.

10. C. A. Rogers and S. J. Taylor, Functions continuous and singular with respect to a Hausdorff measure, Mathematika 8 (1961), 1-31.

11. D. Sullivan, Entropy, Hausdorff measures old and new, and limit sets of geometrically finite Kleinian groups, Acta Math. (to appear).

12. S. J. Taylor, The exact Hausdorff measure of the sample path for planar Brownian motion, Proc. Cambridge Philos. Soc. 60 (1964), 253-258.

13. On the connection between generalized capacities and Hausdorff measure, Proc. Cambridge Philos. Soc. 57 (1961), 524-531.

14. __ Exact asymptotic estimates of Brownian path variation, Duke Math. J. 39 (1972), 219-241.

15. S. J. Taylor and J. G. Wendel, The exact Hausdorff measure of the zero set of a stable process, $\mathrm{Z}$. Warsch. Verw Gebiete 6 (1966), 170-180.

16. S. J. Taylor and C. Tricot, Packing measure of rectifiable subsets in the plane (in preparation).

17. C. Tricot, Sur la classification des ensembles boréliens de mesure de Lebesgue nulle, Thèse de doctorat, Genève, 1979.

18. ___ Douze définitions de la densité logarithmique, C. R. Acad. Sci. Paris 293 (1981), 549-552.

19. T Two definitions of fractional dimension, Math. Proc. Cambridge Philos. Soc. 91 (1982), $57-74$.

Department of Mathematics, University of Virginia, Charlottesville, Virginia 22903

Department of Mathematics, University of British Columbia, Vancouver, British Columbia, CANADA V6T 1 Y4 